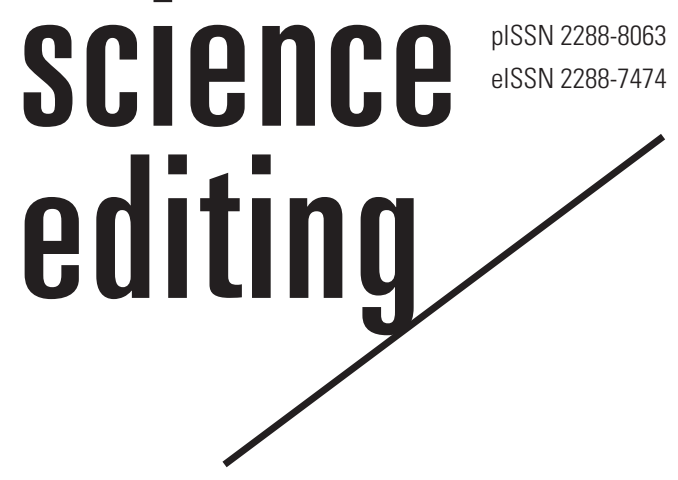

\title{
Comparative analysis of manuscript management systems for scholarly publishing
}

\author{
Soon Kim', Hyungwook Choi ${ }^{2}$, Nayon Kim², EunKyung Chung², Jae Yun Lee ${ }^{3}$ \\ ${ }^{1}$ Research Institute for Social Science, ${ }^{2}$ Department of Library and Information Science, Ewha Womans University, Seoul; \\ ${ }^{3}$ Department of Library and Information Science, Myongji University, Seoul, Korea
}

\section{Abstract}

To improve scholarly communications with scientists throughout the world, an international-level manuscript management system is indispensable. We analyzed the manuscript management systems currently in use in Korea and suggested ways to improve these domestic systems through benchmarking with representative overseas systems. Drawing information from the manufacturer's documentation, we compared the functionalities of the major manuscript management systems available in Korea to international systems. Based on this analysis, we identified the essential elements necessary to meet international standards. The representative international systems provide an intuitive interface and an efficient communication channel for authors, editors, and reviewers, enabling them to save time. The two domestic paid systems are almost at the international level; however, the free systems developed in Korea need to be upgraded. In particular, more advanced visualization tools, more efficient tools for communication between stakeholders, and convenient linking to external content are needed. Studies of these manuscript management systems, which are essential for the internationalization of domestic journals, can be utilized as primary materials to improve the level of Korean academic journals in response to the rapid changes in modern scholarly communication.

Keywords

Journal management system; Manuscript management system; Peer review system; Scholarly

\section{Introduction}

Journal management is a complicated and time-consuming task, and considerable investments of time and workforce must be made to follow traditional journal management procedures. As academic publishing has transitioned into the internet age, journal editors are under great pres- 
sure to shorten the time from manuscript submission to publication. In order to do so, it is important to select a manuscript management system that can effectively manage the review process of journal articles [1].

An online manuscript management system can be used to promptly send and receive manuscripts online and to track the progress of manuscripts from submission to publication. It provides an efficient communication channel for authors, editors, and reviewers, including automated e-mail alerts and convenient visual displays of the peer-review status of submissions [2].

In recent years, many Korean academic societies have used an online manuscript management system provided by the government free of charge. However, despite the great expectations that accompanied this initiative, they have expressed many complaints about the technical incompleteness and complicated interface of this systems [3].

This manuscript management system, which is provided free of charge by governmental agencies, is unable to effectively address the challenges faced by existing users due to difficulties in securing a budget for improving its performance [4]. Therefore, many Korean academic societies have started using a paid foreign or domestic manuscript management system instead of the free system.

To investigate this trend, this study examined and analyzed representative major free and paid manuscript management systems in Korea and abroad, and through a comparative analysis, identified the essential features necessary for establishing an online manuscript management system that meets international standards in a way that is suitable given domestic societies' limited budget.

\section{Methods}

We compared the major functionalities of representative free and paid manuscript management systems available in Korea. As the websites of foreign companies provided quite detailed information, we referred to information available on those sites. However, due to limitations in the information available for the domestic systems, we verified our results through an email interview with the two domestic system vendors (M2community and Medrang/Inforang). We also verified our final result with the representative of ACOMS, Editorial Manager and Scholar One via email.

Table 1 summarizes the manuscript management systems that are most widely used in Korea and abroad.

\section{Results}

\section{International free manuscript management systems Bepress}

bepress (https://www.bepress.com) is a journal publishing system that was launched in 1999 by law professors at UC

Table 1. Comparison of international and domestic manuscript management systems

\begin{tabular}{|c|c|c|c|}
\hline Manuscript management system & Provider & $\begin{array}{l}\text { Estimated no. of } \\
\text { journals using } \\
\text { the system }\end{array}$ & Remarks \\
\hline \multicolumn{4}{|l|}{ Overseas free system } \\
\hline Bepress (Berkeley Electronic Press) & UC Berkeley & 500 & $\begin{array}{l}\text { Mainly used by university publishers and libraries } \\
\text { was acquired by Elsevier in August } 2017\end{array}$ \\
\hline Open Journal Systems & $\begin{array}{l}\text { Public Knowledge Project administered } \\
\text { by } 6 \text { universities, including Stanford University }\end{array}$ & 9,450 & Mainly used by nonprofit organizations worldwide \\
\hline \multicolumn{4}{|l|}{ Overseas paid systems } \\
\hline ScholarOne & Clarivate Analytics & 7,000 & $\begin{array}{l}\text { Used in Wiley, T\&F, Sage, American Chemical Soci- } \\
\text { ety, Emerald, Royal Society of Chemistry etc. }\end{array}$ \\
\hline Editorial Manager & Aries Systems & 6,700 & Used by Springer Nature, T\&F, PLOS, etc. \\
\hline EVISE & Elsevier & 2,000 & Used by Elsevier itself \\
\hline \multicolumn{4}{|l|}{ Domestic paid systems } \\
\hline eSS@i & Medrang/Inforang & 160 & Medicine, basic science, etc. \\
\hline EMS & M2community & 120 & Medicine, etc. \\
\hline
\end{tabular}

ACOMS, Article Contribution Management System; JAMS, Journal \& Article Management System; eSS@i, E-Submission Solution at Inforang. 
Berkeley, including Robert Cooter. It provides Digital Commons, which is a paid publishing solution. As of July 2018, this system is used by approximately 500 institutions, primarily university libraries and university publishers. It was acquired by Elsevier in August 2017 and continues to provide value-added services in conjunction with Elsevier's Scopus or Plum Analytics, while maintaining the current pricing model.

Many professors want to publish journals in their field of study, but commercial publishers are reluctant to publish journals in new or specialized fields. In response to these customers' needs, Digital Commons supports faculty and students to publish their journals through institutional repositories.

It provides a flexible workflow, including double-blind peer review and detailed usage and citation analysis reports for authors and editors. It provides detailed trends of search keywords, searches by region, number of readers by topic, usage trends over time, and so on. This system supports datasets and streaming media, and seeks to improve the visibility and influence of journals through search engine optimization via Google Scholar. It also offers flexible subscription options, such as open access or paid subscriptions.

\section{Open journal systems}

The Open Journal Systems (OJS, https://pkp.sfu.ca/ojs/) is an open journal management and publishing system developed by the Public Knowledge Project through its federally funded efforts to expand and improve access to research. From submission of manuscripts to online publishing and indexing, it supports all stages of the peer review process. It also provides online publishing and management of all content and a flexible subscription model. A separate publishing process can be set up according to the user's requirements and can be installed and used on a user's server [5].

OpenJournalSystem.com which was launched in 2013, has been providing the journal hosting service to the academic societies and institutions to establish online journals using the free OJS software with fee around $\$ 460$ to $\$ 900$ per year. The service is divided into OJS hosting, customization, support, upgrade, training, programming, and editorial services. OJS accommodates various file formats, such as HTML, PDF, EPUB, audio, and video. It provides not only an English-language homepage, but also article search and archiving functions. It integrates the latest publishing technologies such as Google Analytics, so even novice editors can publish journals easily.

According to '2012 Library publishing services: strategies for success', OJS was the most popular system among the survey respondents, accounting for $56.8 \%$ of responses, and approximately $25 \%$ of the libraries had contracts with Berkeley Electronic Press [6].

\section{International paid systems}

\section{ScholarOne}

ScholarOne Manuscripts (https://clarivate.com/products/ scholarone/) is a manuscript management system used by leading commercial publishers, academic societies, associations, and university publishers such as Wiley. It provides an overview of the manuscript status and identifies bottlenecks where the peer review is stagnant so that the review process can proceed smoothly. It allows editors to assign tasks, sends e-mail alerts, and facilitates easy web-based searching. It can capture data and files in multiple languages, and files can be transferred directly into PDF and HTML proofs.

Editors can obtain a complete overview report in a standard format, or can easily create their own reports. It also provides an intuitive system that allows editors to focus on quality work that increases the value of content by reducing the administrative burden. Furthermore, it allows editors to search for reviewers through their internal databases or external sources such as Web of Science. Also, it provides an automated reviewer recommendation tool (Reviewer Locator) using Web of Science data.

Authors can check all the journal-specific requirements, such as file format and size limitations, when submitting a paper, which supports the prompt submission of manuscripts.

\section{Editorial Manager}

Editorial Manager (https://www.ariessys.com/) is a commercial manuscript management system which is now used by many publishers such as Wolters Kluwer, Springer Nature, BMC, Taylor \& Francis, PLOS, etc. Authors benefit from easy, timesaving submission features like Xtract, which uses automated metadata extraction to prepopulate submission fields using the author's uploaded manuscript document. Authors can also use ORCID Single Sign On to login to multiple Editorial Manager sites using only their ORCID ID. Authors have the ability to send their submission directly from a preprint server or from commonly used manuscript composition applications such as Overleaf or ManuscriptsApp, which is possible to submit a manuscript by capturing metadata or files easily, and the reviewers can accept or reject a request for review through a secure deep link without logging in. Integrations with partners like StatReviewer allow editors to share automated statistical analysis with reviewers. ORCID reviewer recognition can be set up to automatically deposit peer review activity to reviewer ORCID profiles.

For the editors, they can use the similarity check, and the artwork quality check automatically. The references of the submitted manuscript are automatically linked to CrossRef and PubMed and reformatted according to the style of the submitted journal. It could save time searching for reviewers 
by interacting with ProQuest's Pivot database. It is possible to conveniently transmit rejected manuscripts and reviewers' comments to cooperating journals. Enterprise Analytics Reporting provides a quick visualization of key data such as manuscript submission status by region.

Completed manuscripts and supplement materials can be exported to a publishing production tracking system such as Production Manager or exported to an evaluation system such as PRE (Peer Review Evaluation). It is possible to manage the user authority by dividing the detailed role of the editor such as editor-in-chief, editor, associate editor, and administrator. RightsLink and other e-commerce platforms can be used to handle article process charges and interoperability Editorial Manager supports integration with standard systems such as ORCID, Ringgold, Funder Registry, JATS, and CRediT.

\section{Elsevier EVISE}

The EVISE system (https://www.elsevier.com/editors/evise) is an online review system developed by Elsevier. With a single sign-on for all Elsevier products, a user can easily access Scopus or ScienceDirect without using a separate login while using EVISE. The system is designed intuitively and adapts to the user's requirements and usage patterns, providing a visual overview so that editors can easily recognize what they need to do, and it is convenient to manage manuscripts under review.

Editors can search for, invite, and manage reviewers with minimal clicks on a single screen. When a new manuscript is received, a similarity check is automatically performed, which allows the editor to determine straightforwardly whether an article has been plagiarized. Editors can create and maintain author-specific customized notices used to notify authors of the review results. The editor can also use features to support and manage special calls. Editors can filter reviewers by invitation status and choose between "all reviewers" or "my reviewers." If a reviewer's email is bounced back, a warning message will be displayed when searching for this reviewer later. If a revised manuscript is reviewed again, information about the previous reviewer is shown. The editor can use Elsevier's new subject categories (OmniScience) and Scopus' discovery tool to search for reviewers that match the topics of interest and search the full EVISE reviewer database. References are automatically linked through Crossref, allowing editors and reviewers to verify the bibliographic records and to ensure that references are entered correctly.

The manuscript submission process is simplified into 4 steps: inputting manuscript information, uploading files, providing additional information, and submitting. Authors' submission files are automatically sorted by the file format, which allows authors to submit articles faster and more easily. Authors can send emails through a simple link to the journal ed- itor in EVISE, facilitating communication between authors and the journal editor. If authors and coauthors log in with their ORCIDs in EVISE, the publication will automatically be added to their ORCID account after publication.

\section{Comparison of international manuscript management systems}

For the authors' convenience, international systems allow use of individual author information, such as ORCID. When submitting a manuscript, the author can specify a co-author, depending on the role of the contributor. The author can either enter the keywords for the manuscript directly or select them from the keyword-controlled database provided by the journal. In the case of EVISE and Editorial Manager, the author can verify the manuscript status before completing the article submission. When submitted manuscripts pass the review process, the review results and editors' comments can be sent to the authors. The author can respond to the reviewers' feedback, which is then passed on to the reviewers or editorial staff automatically.

When reviewers are requested to conduct a peer review, they can indicate whether they intend to do so. Additionally, if they decline the proposed review request, they can appoint a substitute. Authors may request or reject specific reviewers with detailed reasons. When re-uploading a manuscript with the with the reviewers' comments, the file is automatically anonymized to protect the anonymity of the reviewers.

In the Editorial Manager system, reviewers can see the references in the submitted article, which can be automatically accessed through PubMed and Crossref and can be reformatted to fit the journal's reference style.

For editors, articles in a specific stage of the review process can be identified by color or sorting by the peer review status. The editor can conduct similarity checks and send an invitation e-mail by registering a specific reviewer. If the first reviewer refuses the review, the next reviewer will automatically be invited. Moreover, the editor can decide whether to accept or reject submitted papers regardless of their peer-review status. In Editorial Manager, the editor can evaluate reviewers' performance and check their activities for other journals.

Korean free manuscript management systems

\section{Article Contribution Management System}

Since 1997, the Korea Institute of Science and Technology Information (KISTI) has provided the Article Contribution Management System (ACOMS, https://acoms.kisti.re.kr/) to Korean academic societies free of charge. By utilizing bibliographic information and full texts obtained through this system, KISTI shortens the distribution cycle of scholarly content produced by domestic researchers and provides cost savings for database construction, such as the National Digital Sci- 
ence Library and KoreaScience, which provides high-quality English academic journals produced in Korea [7]. As of May 2018, 143 academic journals used ACOMS 4.0 for their English-language website, to manage submissions and the peerreview process, and to link to electronic journal sites.

Authors can indicate when they are not available and can check on the manuscript's progress at every step from submission to publication. Editors can send an automated e-mail whenever they receive a new manuscript. If there is no response from the invited reviewers, the editor can cancel the invitation or send a reminder e-mail. It is also possible to check for papers whose review has been delayed for a specific period.

Meanwhile, ACOMS 4.0, which was developed by KISTI, has been transferred from the the National Research Foundation of Korea and used in 23 journals, most of which are English-language journals, under the name of ACOMS NRF. ACOMS provides many excellent functions, but unlike OJS, which makes open-source software public and enables customization, ACOMS suffers from operational difficulties regarding the choice of which structure(s) to customize based on the needs of each society. Therefore, it is desirable only to provide a mandatory/hidden setting for the standard functions, meaning that the necessary banners and titles are to be produced directly by the society, utilizing only the standardized template function, as in OJS. ACOMS 4.0 version minimizes customization by applying standard template of journal website [8]. Functions that differ from one society to another must be supported through the development of the necessary systems using the provided API function. It is also possible to maintain consistency by not allowing the terminology to be revised for each society, and the Korean/English basic menu uses standard terms as much as possible [8].

\section{Journal \& Article Management System}

The National Research Foundation of Korea has developed an online journal submission and review system called the Journal \& Article Management System (JAMS, https://portal.jams. or.kr/), which provides a system that supports the entire process of publishing journals, including manuscript submission, peer review, and electronic publishing. As of May 2018, 1,189 journals are using this system. It operates in conjunction with the Korea Citation Index, which was developed for quantitative evaluation of domestic journals. It also provides online full-text searching and a preprint service for accepted papers. JAMS 2.0 provides various administrative functions necessary for the operation of academic societies, such as the management of the society homepage, conference organization, and an electronic journal service.

\section{Korean paid systems}

\section{EMS}

M2community provides more specialized online and mobile services (http://www.m2community.co.kr/). Since 2003, it has provided a comprehensive range of services, including manuscript submission/review, publication, and distribution for about 100 academic societies. This service includes modules for PDF auto-conversion, reference extraction and a full-text linking service, and an automated similarity check service. It also supports individual customization for each journal, an EPUB system linked with the journal homepage, and a professional proofreading and printing module.

The advantages for authors include the ease of creating and signing the copyright agreement, inputting their ORCID, sorting the uploaded files according to their preferences, converting files in multiple formats to PDF files, viewing the PDF files before final submission, selecting preferred or excluded reviewers, and the tracking the progress of submitted papers.

Reviewers can see all other reviewers' comments and confirm the author's response to the reviewer's feedback. Editors can verify authors' responses to feedback from reviewers and check all submitted files at each stage. Regardless of peer-review status, editors can decide whether to accept a submitted manuscript and can visualize at a glance the review status of each reviewer.

\section{E-Submission Solution at Inforang}

The E-Submission Solution at Inforang (eSS@i, http://www. medrang.co.kr/) by Medrang/Inforang is a platform that integrates the online submission and review process. It provides one-stop service from manuscript submission to peer-review status at a glance. It also provides various functions, such as preventing delays in the review process, management of publication fees, and statistics for each peer-review stage. It supports effective journal management and circulation by linking with Crossref and ORCID.

Authors can create a copyright agreement, select a preferred reviewer, and sort the uploaded files according to the author's preference. It is possible to convert files in various formats into PDF files and check the PDF files before the final submission. Reviewers can search by title or keyword in PubMed or KoreaMed, and they can see the author's response to the reviewer's feedback immediately. On the editorial side, it is possible to convert an editor's role to an author or a reviewer without requiring a separate account, and editorial decisions can be made about a submitted manuscript regardless of the progress of peer review.

Recently, support has been added for submissions in various formats used in basic science, such as TeX file conversion through arXiv.org ID. It also provides unregistered ORCID veri- 
fication and a linking service with the FundRef institution code.

\section{Comparative analysis of domestic manuscript}

management systems

We analyzed the domestic systems from the authors', reviewers', and editors' points of view. First, authors can register on their own or can be registered by the editorial office, and duplicate checking is possible. An author may designate a coauthor as a corresponding author. In addition, authors can select keywords and metadata for submitted papers within a word limit. The manuscript can be returned by a reviewer or based on the decision of an editor, and it is not necessary to create new metadata if a revised manuscript is submitted.

EMS by M2community (M2-EMS) can input personal identifiers such as ORCID, ISNI (International Standard Name Identifier), PubMed, and Scopus author ID, and can specify the first author and the corresponding author separately, thus making author identification straightforward. M2EMS and eSS@i can upload and convert files in various formats, convert PDF files, guide PDF verification before the final submission, and arrange the uploaded files according to the author's preferences. ACOMS provides several convenient functions, such as designating the first author and the corresponding author separately, easy keyword selection, and choosing preferred reviewers.

Reviewers can usually register on their own or be registered by the editor. If a review request is received, the recipient may express his or her intention to accept or refuse. Reviewers can submit their opinions using the provided online evaluation form and use the temporary save function before submitting their comments. Reviewers can upload attachments in addition to the review results. In ACOMS, they can decide whether to accept or reject a review request without logging in.

Reviewers using M2-EMS and eSS@i can see the authors' responses, and those using M2-EMS can see all comments from authors, reviewers, and editors. In ACOMS, reviewers can indicate dates when they are not available during registration.

Editors can distinguish folders or specific items that require attention based on color-coding. Furthermore, editors can search for reviewers by name, address, institution, subject field, manuscript keyword, or dates of availability. If the preferred reviewer is not yet registered, the editor can easily register the reviewer and request a review. Editors can conveniently visualize the status of the entire review process by obtaining a status report. Moreover, editors can sort manuscripts by the editorial decision (acceptance, rejection, withdrawal, transfer) and upload/download/edit attachments for submitted manuscripts. ACOMS provides the screening status by user authority, so that the editor-in-chief, editors, reviewers, and journal manager can check the submission and review status of manuscripts submitted to the journal.

Furthermore, M2-EMS can display and print the entire report before a reviewer's opinion is submitted, and ACOMS provides a function for sharing opinions among the editors. Within M2-EMS and eSS@i, users can switch roles among reviewers, editors, and authors without logging out.

Analysis of essential factors for the establishment of a new system based on a comparison of online manuscript management systems

Representative international online manuscript management systems provide an efficient communication channel for authors, editors, and reviewers, including various convenient functionalities. Notably, they offer an intuitive interface that saves time through tools such as automated e-mail alerts, standardized templates, and various visualized reports. The 2 domestic paid systems almost meet international standards; however, the domestic free systems need to be upgraded. More advanced visualizations, more efficient communication tools for stakeholders, and convenient linking to external content are needed.

We classified recommendations for improvement for the functionalities available to authors, reviewers, and editors based on JAMS, which is provided by the NRF of Korea free of charge, and is the most common system used by Korean societies. Table 2 shows a comparative analysis of these systems from the authors' point of view.

The author submission process can be simplified to 4 steps: entering manuscript information, uploading files, entering additional information, and submitting the manuscript. Authors' submission files are automatically sorted according to the file format, allowing authors to submit manuscripts more easily and quickly, and authors can e-mail editors through simple links, which supports easy communication. Table 3 presents recommendations for improvements in the functionality available to authors.

It is also important to be able to easily customize the authority and role of the editor-in-chief, journal manager, and editors according to the internal environment of individual societies. Depending on access authority, 4 modules should be developed for authors, journal managers, editors, and reviewers, and different functions should appear after logging in to each module. Depending on the system, modules can be distinguished for convenience, but in practice, each module interworks with the others.

Table 4 shows a comparative analysis of these systems from the reviewers point of view and Table 5 shows recommendations for improvements in the functionalities available to reviewers.

Table 6 shows a comparative analysis of these systems from 
Table 2. Comparative analysis of manuscript management systems from the authors' point of view

\begin{tabular}{|c|c|c|c|c|c|c|c|}
\hline \multicolumn{4}{|c|}{ International } & \multicolumn{4}{|c|}{ Korean } \\
\hline EVISE & ScholarOne & $\begin{array}{c}\text { Editorial } \\
\text { Manager }\end{array}$ & OJS & EMS & eSS@i & ACOMS & JAMS \\
\hline
\end{tabular}

\section{Author registration}

Authors can register themselves on the system or can be pre-registered by the editorial office.

Authors can enter personal identifiers such as ORCID, ISNI, PubMed Author ID, Researcher ID, and Scopus Author ID (as permitted by the journal).

The system supports secondary fields for author information so that international authors can enter alternative names or translated addresses.

Author registrations are checked for duplicates.

\section{Authority control}

Submitting author can designate a co-author to be the corresponding author.

First author and corresponding author can be designated separately.

\section{File uploading}

Author's uploaded files can be automatically sequenced according to publication-defined preference.

Authors can upload single or multiple files in a single compressed (ZIP) format.

Authors can conveniently categorize multiple uploaded files (e.g., images).

Authors can upload a wide variety of file formats for conversion into a PDF file (Word, WordPerfect, Text, RTF, TeX, EPS, LaTex, PDF, GIF, JPEG, TIFF, PowerPoint, Excel, OpenOffice, etc.).

Authors can upload one or multiple files using drag-and-drop.

The author sees interactive feedback about submitted files.

Authors are required to check the PDF version before final submission to the editorial office.

\section{Input keyword}

Authors can select their own keywords that describe the submission.

Authors can select their own keywords that describe their area of expertise.

\section{Input metadata}

Authors have a clear and easy way to submit a revised manuscript without having to resubmit all metadata and files.

Author-entered submission metadata (e.g. abstract, title, short title, etc.) can be limited by a number of characters or words.

\section{Review process communication}

Authors can suggest or oppose reviewers during manuscript submission.

Submission can be bounced back to author for re-submission following inspection and/or editing by the editorial office.

Other authors of a submission may be asked to verify that they contributed to the paper.

OJS, Open Journal Systems; eSS@i, E-Submission Solution at Inforang; ACOMS, Article Contribution Management System; JAMS, Journal \& Article Management System.

a) JAMS's weak points. 
Table 3. Recommendations for improvements in the functionalities available to authors

\begin{tabular}{lc}
\hline Classification & Detailed description of the recommended features \\
\hline Convenience & If an author has a problem using an official email address when registering his or her email, the author can also register an \\
alternative e-mail. & When registering as a new user, desired author information such as ORCID registration should also open in a new window \\
through linkage with the ORCID API, so that ORCID registration can be done automatically. \\
If the author and coauthor log in through ORCID, the article should be added automatically to the author's ORCID account af- \\
ter the article is published. \\
A menu should be configured to upload supplementary files, such as research data and data sets, for research data sharing. \\
If the research is supported by research funds, information should be added through FundRef.
\end{tabular}

ORCID, Open Researcher and Contributor ID; API, application program interface.

Table 4. Comparative analysis of manuscript management systems from the reviewers' point of view

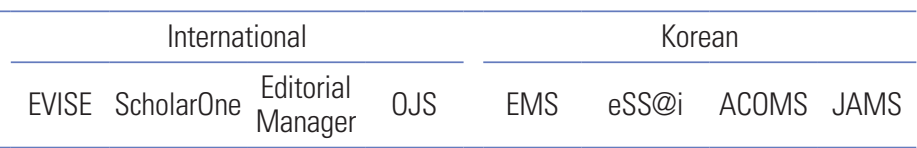

\section{Review process}

Users can register themselves on the system and indicate their

willingness to be reviewers or can be registered by an editor.

Reviewers can accept or decline a reviewer invitation.

Reviewers can respond to reviewer assignments without needing to explicitly log into the system, by clicking on secure links embedded in emails sent to them by the publication.

Reviewers can be prompted to suggest alternate candidate reviewers

if they decline an assignment.

Reviewers can indicate dates when they are not available.

\section{Convenience of a review process}

Reviewers can submit their review online.

Reviewers can save their review for later submission.

Reviewers can use a pre-defined reviewer form.

When reviewing a revised manuscript, reviewers have access to prior revisions of the author's manuscript, with the revision number appended to the PDF file name to allow easier differentiation between versions

Reviewers can view the author's response to their review, which is recorded by the system.

References of manuscript can be automatically linked to PubMed and Crossref, and reformatted to the journal's preferred style.

\section{File uploading}

Reviewers can upload attachments with their recommendations.

Reviewers' uploaded Word and PDF file properties are automatically sanitized to ensure reviewer anonymity.

\begin{tabular}{|c|c|c|c|c|c|c|c|}
\hline 0 & 0 & 0 & 0 & 0 & 0 & 0 & 0 \\
\hline 0 & 0 & 0 & 0 & 0 & 0 & 0 & 0 \\
\hline- & 0 & 0 & - & 0 & 0 & 0 & -a) $^{\text {a) }}$ \\
\hline 0 & 0 & 0 & 0 & - & 0 & - & $-^{\text {a) }}$ \\
\hline- & 0 & 0 & 0 & - & 0 & 0 & -a) $^{\text {a) }}$ \\
\hline 0 & 0 & 0 & 0 & 0 & 0 & 0 & 0 \\
\hline O & 0 & O & 0 & 0 & 0 & 0 & 0 \\
\hline 0 & 0 & 0 & - & 0 & 0 & 0 & 0 \\
\hline- & 0 & 0 & - & 0 & 0 & - & $-^{\text {a) }}$ \\
\hline 0 & 0 & 0 & 0 & 0 & 0 & - & 0 \\
\hline- & - & 0 & - & 0 & - & - & -a) $^{\text {a) }}$ \\
\hline O & 0 & O & 0 & 0 & 0 & 0 & 0 \\
\hline 0 & 0 & 0 & 0 & - & - & - & $-^{\text {a) }}$ \\
\hline
\end{tabular}

OJS, Open Journal Systems; eSS@i, E-Submission Solution at Inforang; ACOMS, Article Contribution Management System; JAMS, Journal \& Article Management System.

a) JAMS's weak points.

Table 5. Recommendations for improvements in the functionalities available to reviewers

\begin{tabular}{ll}
\hline Classification & Detailed description of recommended features \\
\hline Convenience & Allows the reviewers to specify their preferences through searching for their preferred fields. \\
If a reviewer has not responded to a request after the initial invitation, it will automatically send a reminder e-mail. \\
Allow the invited reviewers to accept or reject a review request through a secure deep link without logging in. \\
In case of rejection, the reviewer will be able to recommend other reviewers to replace him/her. \\
The reviewers can make an overall review report online, and the review opinion can be written in a separate file so that the au- \\
thor or the editor can select to view it. \\
$\begin{array}{l}\text { When authors upload a revised manuscript, the reviewers can check the author's responses in the 'Response to Reviewer'. The } \\
\text { final review report can be sent to the editorial committee. } \\
\text { If a revised manuscript is reviewed again, information from the previous editorial committee and the reviewer(s) will be shown. }\end{array}$ \\
\hline
\end{tabular}


Table 6. Comparative analysis of manuscript management systems from the editors' point of view

\begin{tabular}{|c|c|c|c|c|c|c|}
\hline \multicolumn{3}{|c|}{ International } & \multicolumn{4}{|c|}{ Korean } \\
\hline EVISE ScholarOne & $\begin{array}{l}\text { Editorial } \\
\text { Manager }\end{array}$ & OJS & EMS & eSS@i & ACOMS & JAMS \\
\hline
\end{tabular}

\section{Selecting and communicating with reviewers}

Editors can view and manage contributor identifiers such as ORCID, ISNI, Researcher ID, PubMed author ID and Scopus ID.

Editors can search for candidate reviewers by creating custom searches that combine name, address, institution, classifications, manuscript keywords, or other criteria including available dates.

Editors can easily register and invite unregistered users to review a particular manuscript.

Editors can search and invite reviewers from the reviewer discovery database.

Editors can select a group of candidate editors based on keyword matching and can automatically invite them to handle the submission according to configured schedule and preference.

When searching for reviewers, editors can see reviewer activity on other co-operating journals.

Editors can quickly and conveniently invite reviewers who have been suggested or opposed by the submitting author (Appropriate warnings appear during Reviewer searching and selection).

Editors can easily identify manuscripts where reviewers have not yet responded to their invitation, as well as reviews in progress.

Editors can select alternate reviewers who will be automatically promoted and invited if primary reviewers decline the reviewer invitation (configurable option).

Editors can rate reviewer performance.

Editors can view manuscripts with their prior decisions, grouped by decision (accept, reject, withdrawn, transferred).

\section{Peer review process}

"Submit as an Editor" functionality allows editors to upload manuscripts quickly intended for publication without peer review.

Editors can make decisions concerning manuscripts at any time regardless of reviewer status.

Editors can save their comments for later submission.

Editors can display and print a complete layout of their comments before submission.

\section{Convenience of editing}

Editors can search manuscript by manuscript number, author name, keywords, notes, etc.

Editors can quickly display review status as an expandable column that includes detailed information and color coding.

Editors can release an accepted manuscript to an online journal site for pre-print publication (WebFirst).

Editors can upload, download, and edit submission attachments.

Users can switch between reviewer, editor, and author roles without having to log out of the system.

Editors can submit author manuscripts to Similarity Check for plagiarism checking.

Editors can review the results of predictive bibliometrics reports.

\begin{tabular}{|c|c|c|c|c|c|c|c|}
\hline 0 & $\bigcirc$ & 0 & 0 & 0 & 0 & - & -a) \\
\hline 0 & 0 & 0 & 0 & 0 & 0 & 0 & 0 \\
\hline 0 & 0 & 0 & 0 & 0 & 0 & 0 & 0 \\
\hline 0 & 0 & 0 & 0 & 0 & 0 & 0 & 0 \\
\hline O & 0 & 0 & 0 & - & - & - & -a) \\
\hline- & 0 & 0 & - & - & - & - & -a) \\
\hline 0 & 0 & 0 & - & 0 & 0 & - & 0 \\
\hline 0 & 0 & 0 & 0 & 0 & 0 & 0 & 0 \\
\hline 0 & 0 & 0 & - & - & - & - & -a) \\
\hline- & 0 & 0 & - & 0 & 0 & - & -a) \\
\hline 0 & 0 & 0 & 0 & 0 & 0 & 0 & 0 \\
\hline 0 & 0 & 0 & 0 & 0 & 0 & - & -a) \\
\hline 0 & 0 & 0 & 0 & 0 & 0 & - & -a) $^{\text {a) }}$ \\
\hline 0 & 0 & 0 & 0 & 0 & 0 & - & 0 \\
\hline 0 & 0 & 0 & 0 & 0 & 0 & - & -a) $^{\text {a) }}$ \\
\hline 0 & 0 & 0 & 0 & 0 & 0 & - & -a) \\
\hline 0 & - & 0 & - & 0 & 0 & 0 & -a) $^{\text {a) }}$ \\
\hline- & - & 0 & - & 0 & 0 & - & -a) \\
\hline 0 & 0 & 0 & 0 & 0 & 0 & $\underset{\begin{array}{c}\text { (Download } \\
\text { only) }\end{array}}{\triangle}$ & 0 \\
\hline- & 0 & 0 & 0 & 0 & 0 & - & -a) \\
\hline 0 & 0 & 0 & 0 & 0 & 0 & - & 0 \\
\hline- & 0 & 0 & - & 0 & - & - & -a) \\
\hline
\end{tabular}

OJS, Open Journal Systems; eSS@i, E-Submission Solution at Inforang; ACOMS, Article Contribution Management System; JAMS, Journal \& Article Management System.

a) JAMS's weak points. 
Table 7. Recommendations for improvements in the functionalities available to editors

\begin{tabular}{ll}
\hline Classification & Detailed description of recommended features \\
\hline Convenience & Editors can check that the submitted paper meets the aim and scope of the journal and can immediately reject it if needed after \\
judging the worthy of consideration & Editors can easily create and manage author-specific customized announcements to notify authors about a review result. \\
If a specific reviewer's e-mail is bounced back, a warning message is displayed when searching for this reviewer. \\
If the first reviewer rejects a review request within a certain period of time, the invitation email will be automatically sent to the \\
second reviewer. \\
Crossref and Korea Citation Index will automatically link the references so that the editors and reviewers can check the biblio- \\
graphic records and check that the references are entered correctly. \\
Automatically link the submitted manuscript's references to Crossref and Korea Citation Index and reformat them according to \\
the style of the submitted journal. \\
Provide easy searching for reviewers matching topics of interest by linking researcher information from the National Research \\
Foundation of Korea and the Korea Citation Index, such as Scopus's reviewer discovery tools \\
Connecting reviewers' personal profiles (Korean Researcher Information, ResearchGate, Google Scholar, etc.) in order to see the \\
results of the reviewers' research at the invitation of the reviewers, so that the history of the reviewers can be grasped easily. \\
Editors can get a complete overview of the review process and use a standardized report template or easily create reports by \\
themselves. \\
Ouickly visualize and provide key data such as manuscript submission status by region or country. \\
Submitted papers can be divided into new, review, revision, resubmission, and post-decision phases. Papers with an approaching \\
deadline, that are past their deadline, or are new submissions will be displayed in yellow or red for the editor to identify easily \\
Visualization and reporting
\end{tabular}

the editors' point of view and Table 7 shows recommendations for improvements in the functionalities available to editors.

\section{Discussion}

The advent of manuscript management systems has succeeded in achieving dramatic efficiencies in the publishing process, in contrast to traditional publishing procedures. From the 1970s mainframe project to the desktop systems of the 1980s, the advent of the internet in the 1990s, and to Web 2.0 solutions today, the evolving peer review process leverages today's cutting-edge information technology to deliver more effective journal publishing solutions [2].

At a time when scholarly communication is transitioning to an online publishing environment, this study analyzed the current domestic manuscript management systems and suggested ways to improve these systems through benchmarking with representative overseas systems.

A new platform that extends throughout the research life cycle would advance the domestic publishing environment by clarifying the roles of authors, editors, peer reviewers, printing agencies, manuscript editors, and journal managers. The position of the researcher needs to be changed, so that researchers are not only seen as contributors to manuscripts, but also as subjects of research activities who read and write articles.

It is important to design the system intuitively by grasping the user's requirements and usage patterns, to create visual displays that help users to easily recognize what should be done, and to facilitate manuscript management throughout the peer review process.
It is necessary to aim for an open platform that supports recent developments in the peer review process, which is changing to emphasize efficiency and transparency through steps such as post-publication peer review.

Implementing an open research platform that provides support throughout the research life cycle would improve web-based online manuscript management systems and enable them to reach the international level. Domestic journals should prepare an open platform that can support changes in scholarly communication, which is becoming more efficient and transparent.

\section{Conflict of Interest}

No potential conflict of interest relevant to this article was reported.

\section{Acknowledgments}

This work was supported by a research grant from the National Research Foundation of Korea (2017-24 Office of Knowledge and Information Services).

\section{References}

1. Bogunovic H, Pek E, Loncaric S. An electronic journal management system. Paper presented at: 25th International Conference on Information Technology Interfaces; 2003 Jun 19; Cavtat, Croatia. https://doi.org/10.1109/ITI.2003.1225350.

2. Lowrie C, Holmes L. The evolution of web-based peer-re- 
view systems. Learn Publ 2008;21:300-6.

3. Hunter B. Moving open access to open source: transitioning an open-access journal into the open journal systems journal management system. Tech Serv Q 2010;8:31-40.

4. Han JY. The study on implementation of the web-based article submission management system and electronic database in the oceanographic journals. J Korean Soc Inf Manag 2007; 24:343-65. https://doi.org/10.3743/KOSIM.2007.24.1.343.

5. MacGregor J, Stranack K, Willinsky J. The public knowledge project: open source tools for open access to scholarly communication. In: Friesike S, Bartling S. Opening science. Cham: Springer; 2014. p. 165-75.
6. Mullins JL, Rust CM, Ogburn JL, et al. Library publishing services: strategies for success [Internet]. [place unknown]: SPARC; 2012 [cited 2018 Aug 1]. Available from: http:// wp.sparc.arl.org/lps/

7. Jeong HS, Park JW, Lee YS. Design of integrated portal service system for creation of high quality scientific and technologic academic information. J Korea Multimed Soc 2009;12:1530-8.

8. Jung Y. Scholarly publishing platform of KISTI. Presented at: 2018 Korean Council of Science Editors Conference; 2018 Jan 19; Seoul, Korea. 